A.G. Mamalis, S.N. Lavrynenko, A.I. Grabchenko, L.G. Duebner and N.M. Kirjukhin

\title{
Biomedical functional surface generation with control at the nanoscale
}

ABSTRACT. The various processes of manufacturing parts for different bioengineering and medical applications with special physico-chemical attributes at their surfaces and with specific forms and sizes are of great industrial importance. It is essential to create a highly effective process for the generation of these functional surfaces with predetermined macro-, micro- and nanostructures for specific biological applications in the human body. Functional surfaces for biomedical applications can be provisionally divided into two sub-groups: (1) finished nanostructural surfaces after ultraprecision single-point diamond machining; and (2) preliminary surfaces of the workpieces prepared for subsequent coating, which involves generation of the functional surfaces with a coated layer using special materials and technologies. An account of the generation of these functional surfaces for biomedicine in the nanoscale régime is given in this paper.

Nanotechnology Perceptions 1 (2005) 79-87

Nonsubscribers: purchase individual article 